\title{
Cooling system of bearing in high speed feed system
}

\author{
Li Rong1, a, Lin Wei" b, Wang Yanan', c, Zhang Yin ${ }^{1, ~ d, ~ Z h a n g ~ J u y o n g ~}{ }^{1,}$, , Li \\ Chunguang ${ }^{1, f}$ and Chen Zhiping ${ }^{1, g}$ \\ ${ }^{1}$ Hangzhou Dianzi University, Hangzhou, China, 310018; \\ 2Zhejiang University, Hangzhou, China, 310018
}

alirongjx@hdu.edu.cn, b1305713560@qq.com, c1287577260@qq.com, d529358477@qq.com, ezhang_juyong01@163.com, flichunguang@hdu.edu.cn, glichunguang@hdu.edu.cn

\begin{abstract}
Keywords: Finite element analysis; Positioning precision; cooling system
Abstract. In the high speed feed system, the temperature rise of the bearing will affect the positioning accuracy, so we design the liquid cooling system to cool the bearing. The cooling system consists of the mechanical module and the liquid-cycle-cooling module. The mechanical module is a spiral groove on the inner surface of the bearing seat, and the coolant cools the bearing through the spiral groove. The liquid-cycle-cooling module is mainly composed of a cooler, a one-way variable pump and temperature sensor to control the flow rate of the coolant. The temperature rise of the bearing is reduced by using the mechanical module and the liquid-cycle-cooling module, thereby improving the positioning accuracy of the machine tool.
\end{abstract}

\section{Introduction}

The thermal error of the high-speed feed system has become a key factor affecting the machining accuracy and stability of the CNC machine tools. With the increase of the feed speed, the thermal deformation error and the positioning error of the machine tool are increased. The research shows that thermal error has become the largest error source of NC machine tool, which accounts for $40 \%$ to $70 \%$ of the total error [1].

The thermal deformation error caused by the temperature rise is the main part of the positioning error in the feed system [2]. Therefore, the best way to improve the machining accuracy is to reduce the thermal deformation error of the feed system [3]. With the increase of the ball screw speed, the heating of the bearing becomes more serious, which affects the positioning accuracy of the feed system directly. Consequently, it is necessary to propose a liquid cooling system to absorb the heat of the bearing [4].

\section{Cooling scheme}

Due to the high-speed rotation of the ball screw, the frictional torque between the inner and outer rings of the bearing and the ball is increased, resulting in a large amount of heat generated by the bearing [5]. Part of the heat is transferred to the two section of the screw, which leads to the axial thermal deformation of the ball screw. Another part of the heat transfer to the bearing seat, causing thermal deformation of the bearing. Therefore, the thermal deformation error of the bearing is increased, and the positioning precision of the feed system is influenced [6].

The thermal deformation of the ball screw and bearing seat is caused by the bearing heat, so it is necessary to reduce the temperature rise of the bearing. Considering that the bearing transfers heat to the screw and the support through the inner surface and the outer surface [7]. Therefore, the spiral grooves are formed on the mating surfaces of the bearing seat, and the coolant cools the bearing through the spiral groove.

\section{Mechanical module}

In order to avoid the leakage of the coolant into the inner of the bearing, the spiral groove is processed in sections on the inner surface of the bearing seat. Each bearing corresponds to a spiral 
groove, and each end of the spiral groove has an oil intake hole and an oil outlet. The coolant enters the spiral groove from the inlet hole, and then flows out from the oil outlet. The concrete structure of the spiral groove is shown in Fig. 1.

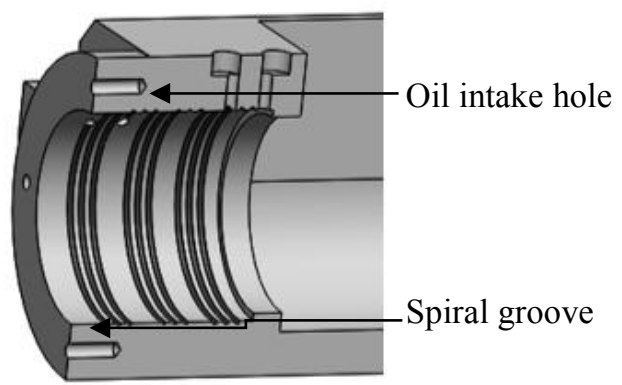

Bearing seat

Fig.1. Structure diagram of spiral groove

Each spiral groove has a cooling circuit, and the cooling circuit does not interfere with each other. When the liquid-cycle-cooling system is working, the coolant enters the oil inlet through the sleeve pipe joint, and then flows through the oil outlet to form a cycle cooling process.

\section{Liquid-cycle-cooling module}

According to the working state of the feeding system, the flow rate of the cooling system can be adjusted. When the speed increases, the heat of the bearing increases, so the flow rate of the coolant increase. When the rotation speed of the ball screw is reduced, the calorific value of the bearing is reduced, so the coolant flow rate are reduced correspondingly.

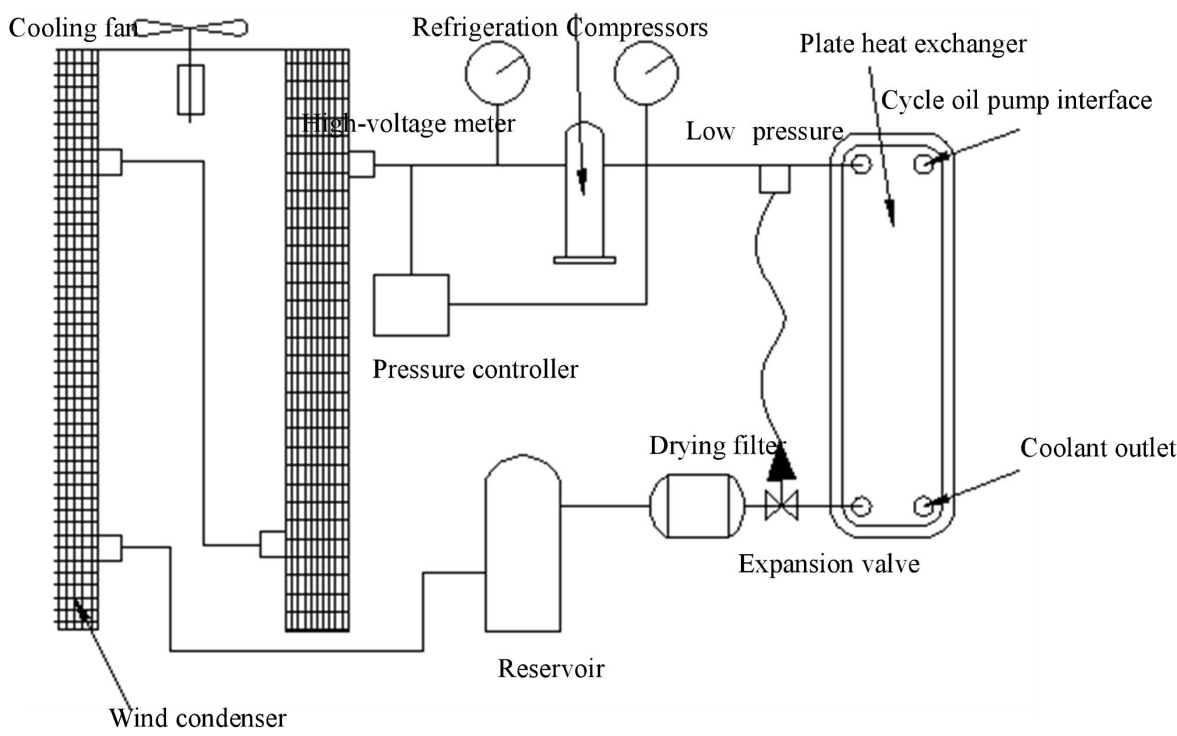

Fig.2. Adjustable liquid-cycle-cooling module

The liquid-cycle-cooling system includes oil pump, fuel tank, cooler, radiator, temperature sensor, throttle valve, tubing and related hydraulic auxiliary components. The flow rate of the coolant can be adjusted by software control. The temperature sensor is used to measure the temperature of the inlet and outlet coolants, and the coolant flow rate is determined according to the temperature difference. The working principle is shown in Fig. 2. Firstly, the high temperature coolant is reused into the circulating oil pump. Later, it is pressurized by the circulating oil pump and then flows into the oil cooler for cooling treatment. After cooling treatment, the feed system is cooled again.

In order to better control the flow rate of coolant, the throttle valve is installed at the outlet of the coolant to control the flow rate. After adjusting the flow rate, the coolant is separated from the branch 
to cool the main heating position. After cooling, the coolant is recirculated into the fuel tank again, thus forming a cycle cooling process.

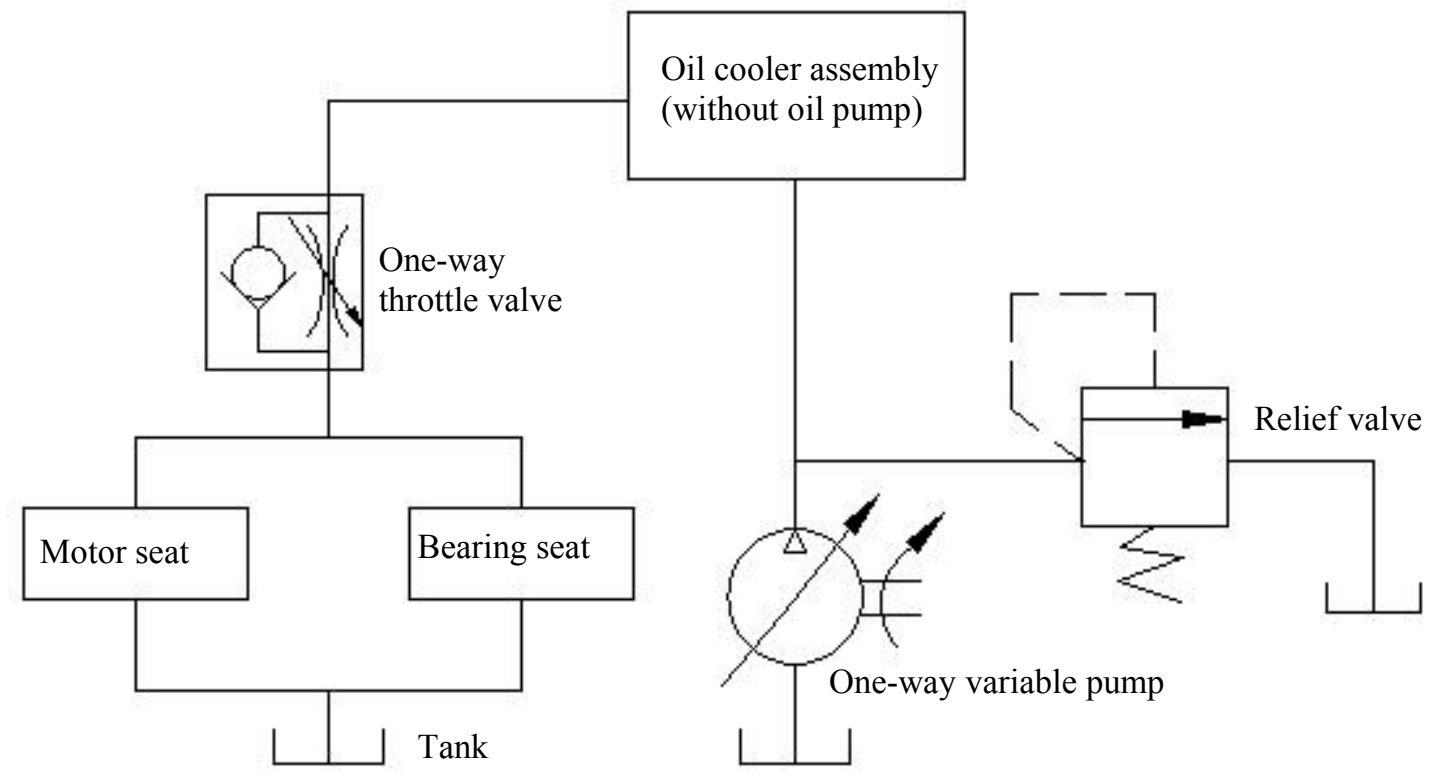

Fig. 3. Liquid-cooled hydraulic circuit

In this circuit, we need to adjust the flow rate of the coolant in real time according to the heat generation of the bearing seat, and the flow rate can be adjusted by controlling the power of the variable pump. The evaluation of heating power depends on the temperature difference of the coolant flowing into the bearing seat and the outflow bearing seat.

The hydraulic circuit of the liquid-cycle-cooling module is shown in Fig. 3, in which the oil pump is a one-way variable pump, and the throttle valve regulates the flow of the coolant. The relief valve plays a role in limiting the oil pump pressure and maintaining the pressure effect.

\section{Conclusion}

This paper introduces the working principle of the liquid cooling system, and introduces the concrete structure of the mechanical module and the liquid-cycle-cooling module. The spiral groove of the bearing seat provides a cooling groove for the bearing to ensure the normal operation of the bearing. The liquid cooling module provides the cooling circuit for the bearing, and then realizes the adjustment of the coolant flow rate to meet different working conditions. Combined with the mechanical module and the liquid-cycle-cooling module, the bearing temperature is reduced, thereby improving the positioning accuracy of the machine tool.

\section{Acknowledgements}

This work was financially supported by the Zhejiang Province Commonweal Projects of China (2015C31077).

\section{References}

[1] Fu Jianzhong, Yao Xinyu, He Yong, et al. The development of thermal error compensation technology for CNC machine tools [J]. Aviation Manufacturing Techology, 2010, (4), 64-66.

[2] Yang Jinbin, Yang Weiping, Huang Guiying. Ball screw thermal displacement suppression measures [J]. Manufacturing Technology and Machine Tools, 2006, (8): 109-110. 
[3] Guo Ce, Sun Qinghong. High-speed high-precision CNC lathe spindle thermal analysis and thermal deformation calculation [J]. Journal of Southeast University: Natural Science Edition, 2007, (2): 37-39.

[4] O. Horejs. Thermo-mechanical Model of Ball Screws with Non-steady Heat Sources [J]. Thermal issues in Emerging Technologies, 2007, (6): 17-19.

[5] Shen Xiaoyan. Theoretical and experimental study on the dynamic characteristics of high speed precision ball screw pair [D]. Nanjing University of Science and Technology, 2017:65-67.

[6] Zou Cunjian, thermal error analysis and experimental study of CNC machine tool feed system [D]. ShenYang Ligong University, 2017:32-37.

[7] Chen Weifu. Analysis and test of bearing thermal characteristics of machine tool feed system [D]. Nanjing University of Science and Technology, 2015:1-6. 